\title{
Enhanced Particle Swarm Optimization for Short-Term Non-Convex Economic Scheduling of Hydrothermal Energy Systems
}

\author{
Vinay Kumar Jadoun ${ }^{\dagger}$, Nikhil Gupta*, K. R. Niazi* and Anil Swarnkar*
}

\begin{abstract}
This paper presents an Enhanced Particle Swarm Optimization (EPSO) to solve short-term hydrothermal scheduling (STHS) problem with non-convex fuel cost function and a variety of operational constraints related to hydro and thermal units. The operators of the conventional PSO are dynamically controlled using exponential functions for better exploration and exploitation of the search space. The overall methodology efficiently regulates the velocity of particles during their flight and results in substantial improvement in the conventional PSO. The effectiveness of the proposed method has been tested for STHS of two standard test generating systems while considering several operational constraints like system power balance constraints, power generation limit constraints, reservoir storage volume limit constraints, water discharge rate limit constraints, water dynamic balance constraints, initial and end reservoir storage volume limit constraints, valve-point loading effect, etc. The application results show that the proposed EPSO method is capable to solve the hard combinatorial constraint optimization problems very efficiently.
\end{abstract}

Keywords: Short-term hydrothermal scheduling, Fuel cost minimization, Particle swarm optimization, Valve-point loading effect, Constriction functions

\section{Introduction}

In the present competitive environment, the short-term hydrothermal scheduling (STHS) plays significant role for economic operation of power systems. The main objective of STHS problem is to schedule the thermal and hydro plants so as to minimize the fuel cost of thermal units while ensuring the optimum use of available water reserves and satisfying several operational and network constraints. STHS is a non-convex, complex combinatorial optimization problem having various operational constraints such as power balance, power generation limits, reservoir storage volume limits, water discharge rate limits, water dynamic balance, initial and end reservoir storage volume limits, valve-point loading effect, etc. The classical optimization methods such as Mixed Integer Programming, Dynamic Programming, Gradient Search Method, Nonlinear Programming, Mathematical Decomposition and Lagrange Relaxation, etc. are not suitable to solve such optimization problems due to their inherent shortcoming in handling non convexity and complex inequalities constraints except dynamic programming. However, dynamic programming suffers from the curse of dimensionality especially in large power systems [1]. In recent years, various Artificial Intelligence (AI) based meta-heuristic approaches such as

$\dagger$ Corresponding Author: Dept. of Electrical Engineering, Malaviya National Institute of Technology, Jaipur, India. (vjadounmnit@ gmail.com)

* Dept. of Electrical Engineering, Malaviya National Institute of Technology, Jaipur, India. (\{nikhil2007_mnit, krn152001\}@yahoo.co. in, mnit.anil@gmail.com)

Received: April 11, 2014; Accepted: June 1, 2015
Simulated Annealing (SA), Differential Evolution (DE), Evolutionary Programming (EP), Genetic Algorithm (GA), Cultural Algorithm (CA) Particle Swarm Optimization (PSO), etc. have been attempted to solve the STHS problem [2]. However, Particle Swarm Optimization (PSO) has several advantages over other meta-heuristic techniques in terms of simplicity, convergence speed, and robustness [3]. It provides convergence to the global or near global point, irrespective of the shape or discontinuities of the cost function [4]. The performance of the PSO greatly depends on its parameters and it often suffers from the problems such as being trapped in local optima due to premature convergence [5], lack of efficient mechanism to treat the constraints [6], loss of diversity and performance in optimization process [7], etc. PSO is a population based meta-heuristic optimization technique in which the movement of particles is governed by the two stochastic acceleration coefficients, i.e., cognitive and social components and the inertia component. In order to enhance its exploration and exploitation capabilities, the components affecting velocity of particles should be properly managed and controlled.

Several methods have been reported in the recent past to enhance the computational efficiency of the conventional PSO. A constriction factor was suggested in the control equation to assure convergence of PSO [8-10]. However, the exact determination of this factor is computationally demanding. Selvakumar and Thanushkodi [11] modified cognitive behavior of the swarm by considering worst experience of the particle. This method provides some additional diversity but showing poor local searching ability 
unless supported by a heuristic local random search algorithm. Roy and Ghoshal [12] proposed Crazy PSO (CPSO) to improve global search and convergence. This method however, requires defining the probability of craziness which can only be achieved after several experimentations. Some attempts [13-16] have been made to vary the cognitive and social behavior of the swarm during the search process by dynamically controlling the acceleration coefficients. Again the determination of limiting values of the acceleration coefficients is a difficult task. Efforts have also been made to suggest a new formulation of the control Eq. (5). These methods require determination of proper values of parameters.

In the light of above discussion a new Enhanced Particle Swarm Optimization (EPSO) method has been proposed to efficiently solve STHS problem of power systems. The proposed method effectively controls and regulates the velocity of particles during their flights so as to ensure global exploration and to facilitate local exploitation. In the following section the formulation for STHS is presented. The proposed EPSO method is explained in section 3. The simulation results are reported in section 4. Finally, the conclusions are summarized in section 5.

\section{Problem Formulation}

The main objective of STHS problem is to minimize the fuel cost of thermal units over the predicted load demand for specified period of time, while satisfying various operational and network constraints. The large turbine thermal generators usually have a number of fuel admission valves which are operated in sequence to meet out increased generation. The opening of a valve increases the throttling losses rapidly and thus the incremental heat rate rises suddenly. This valve-point loading effect introduces ripples in the heat-rate curves and can be modeled as sinusoidal function in the generator cost function. Therefore, the objective function for the STHS problem may be stated as to minimize

$$
F\left(P_{s i t}\right)=\sum_{t=1}^{T} \sum_{i=1}^{N_{s}}\left(a_{i}+b_{i} P_{s i t}+c_{i} P_{s i t}^{2}\right)+\left|e_{i} \sin \left(f_{i}\left(P_{s i \min }-P_{s i t}\right)\right)\right|
$$

where $a_{i}, b_{i}, c_{i}$, are the cost coefficients of the $i$ th generator, and $e_{i}$ and $f_{i}$ are the valve-point effect coefficients of the $i$ th generator, $P_{s i t}$ is the real power output of the $i$ th generator for the $t$ th schedule interval, $P_{\text {simin }}$ is the minimum output limit of the $i$ th thermal plant, $T$ is the total scheduled time interval and $N_{s}$ is the number of thermal generating units in the system. The problem constraints pertaining to hydrothermal systems are briefly described in the following sub-sections.

\subsection{System power balance}

The sum of total power generation of all thermal and hydro generators must be equal to the sum of total power demand plus the network power loss. The network power loss can be evaluated using B-coefficient loss formula [17]. Therefore, the system power balance equation may be stated as

$$
\begin{gathered}
\sum_{i=1}^{N_{s}} P_{s i t}+\sum_{j=1}^{N_{h}} P_{h j t}=P D_{t}+\sum_{k=1}^{N_{s}+N_{h}} \sum_{l=1}^{N_{s}+N_{h}} P_{k t} B_{k l} P_{l t}+\sum_{k=1}^{N_{s}+N_{h}} B_{0 k} P_{k t}+B_{00} \\
i=1,2, \ldots, N_{s} ; j=1,2, \ldots N_{h} ; t=1,2, \ldots, T
\end{gathered}
$$

where, $P_{s i t}, P_{h j t}$, are power generation from $i$ th thermal and $j$ th hydro generator at the $t$ th schedule interval, $N_{s}, N_{h}$ are the respective total number of generators in the system, $P_{D t}$ is the load demand at the $t$ th schedule interval, $P_{k t}$ and $P_{l t}$ are power generation of the $k$ th and $l$ th index of plants in the hydrothermal system, $B, B_{0}$ and $B_{00}$ denote the power loss coefficients.

$$
P_{h j t}=C_{1 j} V_{h j t}^{2}+C_{2 j} Q_{h j t}^{2}+C_{3 j} V_{h j t} Q_{h j t}+C_{4} V_{h j t}+C_{5 j} Q_{h j t}+C_{6 j}
$$

where $Q_{h j t}, V_{h j t}$ are the water release and reservoir storage volume of the $j$ th hydro plant at the $t$ th schedule interval; $C_{1 j}, C_{2 j}, C_{3 j}, C_{4 j}, C_{5 j}$ and $C_{6 j}$ are the power generation coefficients of the $j$ th hydro plant.

\subsection{Power generation limits}

For stable operation, power output of each generator is restricted within its minimum and maximum limits. The generator power limits are expressed as

$$
\begin{gathered}
P_{s i, \text { min }} \leq P_{s i} \leq P_{s i, \text { max }} \\
P_{h j, \text { min }} \leq P_{h j} \leq P_{h j, \text { max }}
\end{gathered}
$$

where $P_{\text {si,min }}, P_{\text {si,max }}$ are the minimum and maximum power output limits of the $i$ th thermal plant; $P_{h j, \min }, P_{h j, \max }$ are the minimum and maximum power output limits of the $j$ th hydro plant.

\subsection{Reservoir storage volume limit}

The reservoir storage volume limit of each hydro plant is restricted within its minimum and maximum limits and is expressed as

$$
V_{h j, \min } \leq V_{h j t} \leq V_{h j, \max }
$$

where, $V_{h j, \min }, V_{h j, \max }$ are the lower and upper bounds of reservoir storage volume limits of the $j$ th hydro plant.

\subsection{Water discharge rate limit}

The water discharge rate limit of each hydro plant is restricted within its minimum and maximum limits and is 
expressed as

$$
Q_{h j, \min } \leq Q_{h j t} \leq Q_{h j, \max }
$$

where $Q_{h j, \min }, Q_{h j, \max }$ are the minimum and maximum water discharge rate limits of the $j$ th hydro plant.

\subsection{Water dynamic balance}

$$
V_{h j t}=V_{h j t-1}+I_{h j t}-Q_{h j t}-S_{h j t}+\sum_{h=1}^{N_{j}}\left(Q_{h t-\tau_{h j}}+S_{h t-\tau_{h j}}\right)
$$

where $I_{h j t}, S_{h j t}$ are the inflow and spillage of the $j$ th hydro plant at the $t$ th schedule interval, respectively; $\tau_{h j}$ is the time delay between $j$ th hydro plant and its upstream $h$ th plant at schedule interval $t ; N_{j}$ is the number of upstream plants directly above the $j$ th hydro plant.

\subsection{Initial and end (terminal) reservoir storage volumes limits}

$$
\begin{aligned}
& V_{j 0}=V_{j B}, V_{j T}=V_{j E} ; j=1,2, \ldots N_{h} \\
& i=1,2, \ldots, N_{s} ; t=1,2, \ldots, T
\end{aligned}
$$

where $V_{j 0}, V_{j T}$ are the reservoir storage of hydro plant $j$ at interval 0 and $T ; V_{j B}, V_{j E}$ are the initial and end (terminal) reservoir storage limits of the $j$ th hydro plant, respectively.

\section{Proposed EPSO}

The conventional PSO is initialized with a population of random solutions and searches for optima by updating particle positions. The velocity of the particle is influenced by the three components: initial, cognitive and the social component. Each particle updates its previous velocity and position vectors according to the following model [18]:

$$
\begin{aligned}
v_{i}^{k+1}=W v_{i}^{k} & +C_{1} \times \operatorname{rand}_{1}() \times \frac{\text { pbest }_{i}^{k}-s_{i}^{k}}{\Delta t} \\
& +C_{2} \times \operatorname{rand}_{2}() \times \frac{\text { gbest }^{k}-s_{i}^{k}}{\Delta t} \\
s_{j}^{k+1}= & s_{j}^{k}+v_{j}^{k+1} \times \Delta t
\end{aligned}
$$

where $v_{i k}$ is the velocity of $i$ th particle at $k$ th iteration, $\operatorname{rand}_{1}(\mathrm{)})$ and $\operatorname{rand}_{2}(\mathrm{)})$ are random numbers between 0 and 1 , $s_{i k}$ is the position of $i$ th particle at $k$ th iteration, $C_{1}, C_{2}$ are the acceleration coefficients, pbest ${ }_{i}^{k}$ is the best position of $i$ th particle at $k$ th iteration achieved based on its own experience, gbest ${ }^{k}$ is the best particle position based on overall swarm experience, $\Delta t$ is the time step, usually set to 1 second and $W$ is the inertia weight which is allowed to decrease linearly as follows

$$
W=W_{\min }+\frac{\left(W_{\max }-W_{\min }\right) \times\left(i t r_{\max }-i t r\right)}{i t r_{\max }}
$$

where $W_{\min }$ and $W_{\max }$ are the minimum and maximum value of inertia weight respectively, $i t r_{\max }$ is the maximum number of iterations and itr is the current number of iteration.

For better performance of PSO, the particles must fly with higher velocities during the early flights to enhance global search and should be gradually slowed down during later flights of the journey to improve local search. This needs a proper balance between cognitive and social behaviors of the swarm. Initially, the impact of cognitive component must be high and that of the social component be less to ensure global exploration of the search space. However, during later part of the journey, the social component must dominate over the cognitive one so as to divert all particles towards the global best to enhance local exploitation. This is essential for maintaining a good balance between exploration and exploitation as suggested by [14]. Therefore, a modified control equation is suggested for dynamically regulating particle's velocity, by suggesting suitable exponential constriction functions $\zeta_{1}$ and $\zeta_{2}$. In addition, the cognitive behavior is split to encompass best and preceding experience of the particle. The suggested control equation for the proposed EPSO may be expressed as

$$
\begin{aligned}
v_{i}^{k+1}= & W \times v_{i}^{k}+\zeta_{1} \times C_{1 b} \times \text { rand }_{1}() \times \frac{\text { pbest }_{i}^{k}-s_{i}^{k}}{\Delta t} \\
& +\left(1-\zeta_{1}\right) \times C_{1 p} \times \text { rand }_{2}() \times \frac{s_{i}^{k}-\text { ppreceding }_{i}^{k}}{\Delta t} \\
& +\zeta_{2} \times C_{2} \times \text { rand }_{3}() \times \frac{\text { gbest }^{k}-s_{i}^{k}}{\Delta t}
\end{aligned}
$$

The modifications suggested in the control equation are explained in the following sub-sections.

\subsection{Inertia weight update}

The inertia weight is employed to control the impact of the previous velocity on the current velocity. Thus, the parameter $W$ regulates the tradeoff between the exploration and exploitation potential of the swarm. A large inertia weight facilitates exploration, while a small weight tends to facilitate local exploitation. A proper value of the inertia weight is one of the deciding factors to obtain better solutions. It is preferable to initially set the inertia weight at large value to promote global exploration of the search space, and gradually decrease it to obtain refined solutions [14]. Shi and Eberhart [18] suggested linear modulations of inertia weight. Normally convergence characteristics of any search techniques follow nearly exponential decay and so it may be intuitively believed that exponential decay of the inertia weight function can provide a better balance 
between the global and local search. Therefore, in the proposed method, the inertia weight has been allowed to vary in accordance with an exponential decaying function rather than to decrease linearly. The modulations suggested to update the inertia weight is governed by the following relation:

$$
W=\exp \left(-\eta \log _{\mathrm{e}}\left(W_{\max } / W_{\min }\right)\right)
$$

where, $\eta=i t r / i t r_{\max } ; i t r_{\min } \leq i t r \leq i t r_{\max }$ and itr is the iteration count which is being varied from $i t r_{\min }$ to $i t r_{\max }$.

\subsection{Updating preceding experience}

In order to improve the diversity, the cognitive behavior was split in [11] by considering the worst experience in addition to the best experience of particles. Although, this modification provides additional diversity but it results in poor cognitive behavior and requires a local random search algorithm to enhance exploitation potential of PSO. Therefore, in the proposed method, the concept of preceding experience is suggested instead of the worst experience to improve the cognitive behavior of the swarm. Here the current fitness of each particle is compared with its fitness value in the preceding iteration, and if it is found less, it will be treated as the preceding experience. The preceding experience of the particle produces much less diversity than the worst particle and thus provides better exploration and exploitation of the search space without any additional local random search or else.

\subsection{Dynamic Control of Acceleration Coefficients}

The cognitive and social behavior plays an important role in searching the global area and global optima. In conventional PSO, these behaviors are governed by static acceleration coefficients. However, many researchers [8-10, 13-16, 19] suggested that these acceleration coefficients must be dynamically controlled with iterations to regulate particle's velocity during the whole computation process but faces difficulty as discussed in section 1 . In the present work, the acceleration coefficients are also dynamically controlled by introducing two exponential constriction functions $\zeta_{1}$ and $\zeta_{2}$ which are defined as

$$
\begin{gathered}
\zeta_{1}=\mathrm{e}^{-\mu_{1} \eta} \\
\zeta_{2}=\kappa \mathrm{e}^{\mu_{2} \eta} ; \kappa=\zeta_{1} \mathrm{C}_{1 \mathrm{~b}} / \zeta_{2} \mathrm{C}_{2}
\end{gathered}
$$

where, $\kappa$ is the ratio of proposed dynamic cognitive and social acceleration coefficients. For identical values of these coefficients at $\eta=\eta_{t}$

$$
\kappa=\left(C_{1 \mathrm{~b}} / C_{2}\right) e^{-\eta_{t}\left(\mu_{1}+\mu_{2}\right)}
$$

Next, for social behavior to be $k_{e}$ at the end of search

$$
\kappa=\left(k_{e} / C_{2}\right) e^{-\mu_{2}}
$$

Thus, from (17) and (18)

$$
\mu_{2}=\left(1-\eta_{t}\right) / \eta_{t} \times\left(\eta_{t} \mu_{1}+\log _{e}\left(k_{e} / C_{1 \mathrm{~b}}\right)\right)
$$

For the given values of $C_{1 \mathrm{~b}}, C_{2}, \mu_{1}$ and $\eta_{t}$, the value of $\mu_{2}$ can be optimized for the desired value of $k_{e}$.

The above mentioned alterations in the control equation of the conventional PSO regulates particles' velocity within predefined bounds without any additional formulation as reported in many improved versions of PSO [4, 5, 7, 9, 12$16]$, yet preserving diversity due to the stochastic nature of cognitive and social behaviors of the swarm.

\subsection{Particle encoding and initialization}

The solution of an STHS problem is the set of most optimal hourly reservoir water discharges and thermal generations over the entire scheduling horizon for the desired objective(s) bounded by certain operational constraints. In the proposed PSO, the particles are encoded in real numbers as the set of current water discharge and thermal generations which is generated randomly within their prescribed minimum and maximum limits. The structure of an individual $P$ consists of $N_{h}$ hydro plants and $N_{s}$ thermal plants for $T$ time intervals considered is shown in Fig.1. The figure shows hourly water discharges of hydro plants and power outputs of thermal plants.

$$
P=\left[\begin{array}{llllllll}
Q_{h 1,1} & Q_{h 2,1} & \ldots & Q_{h N_{h}, 1} & P_{s 1,1} & P_{s 2,1} & \ldots & P_{s N, 1} \\
Q_{h 1,2} & Q_{h 2,2} & \ldots & Q_{h N_{h}, 2} & P_{s 1,2} & P_{s 2,2} & \ldots & P_{s N, 2} \\
\cdot & \cdot & \ldots & \cdot & \cdot & \cdot & \ldots & \cdot \\
\cdot & \cdot & \ldots & \cdot & \cdot & \cdot & \ldots & \cdot \\
\cdot & \cdot & \ldots & . & \cdot & \dot{.} & \ldots & \cdot \\
Q_{h 1, T} & \dot{Q}_{h 2, T} & \ldots & Q_{h N_{h}, T} & P_{s 1, T} & P_{s 2, T} & \ldots & P_{s N, T}
\end{array}\right]
$$

Fig. 1. Particle encoding for the proposed PSO

The initial population is randomly created with predefined number of particles to maintain diversity. Each of these particles satisfies problem constraints defined by Eqs. (2)-(9). Infeasible particle, if appeared, is not rejected but corrected using a correction algorithm as described in section 3.5. This improves the pace of PSO and thus reduces its computation time. The fitness of each particle is evaluated using (1) and then pbest, ppreceding and gbest are initialized. The initial velocity of particles is assumed to be zero.

\subsection{Correction algorithm}

In PSO, the velocity and position update may create infeasible solutions. These infeasible solutions are not rejected but are corrected to feasible ones by using a correction algorithm. In STHS problem, the correction 
algorithm takes care of the initial and end-storage constraints of reservoir and also the system power balance constraint in addition to maximum and minimum generation limits, etc. The end storage volume of any reservoir can be expressed as a function of hydro water discharge, assuming the spillage in Eq. (8) to be zero [20]. For handling the initial and end reservoir storage constraints, a dependent time interval $\mathrm{d}$ is randomly selected, which is not repeated in the next time interval and its discharge is calculated from (20).

$$
\begin{aligned}
& Q_{h}(j, d)=V_{h}(j, \text { initial })-V_{h}(j, \text { end }) \\
& \quad+\sum_{t=1}^{T} I_{h}(j, t)+\sum_{m=1}^{N_{j}} \sum_{t=1}^{T} Q_{h}\left(m, t-\tau_{h} m\right)-\sum_{t=1, j \neq d}^{T} Q_{h}(j, t)
\end{aligned}
$$

After handling the initial and end reservoir storage constraints, the volume of reservoir $\left(V_{h}\right)$ is calculated using (8) and satisfies its limit from Eq. (6). Then based on the available water discharge $Q_{h}$ and volume of reservoir $V_{h}$, the generated power by hydro power plants is calculated using (3), while keeping all the generators within their respective limits. To ensure system power balance constraints, the generations of all thermal generators are adjusted by their respective bounded generation limits and then the error is calculated from the power balance equation. The error in the power is equally distributed among all generators and the procedure is repeated till the error is reduced to a predefined mismatch value $\varepsilon$. In this work, the mismatch is considered as 0.001 . This reduces the computational burden of PSO.

\subsection{Elitism and termination criterion}

In stochastic based algorithms like PSO, the solution with the best fitness in the current iteration may be lost in the next iteration. Therefore, the particle with the best fitness is kept preserved for the next iteration. The algorithm is terminated when either all particles reach to the global best position or the predefined maximum iteration number is reached.

\section{Simulation Results}

The proposed EPSO method has been investigated on two different hydrothermal systems with various operational constraints. The value of acceleration coefficients for the proposed EPSO is taken as 1.6, 0.4 and 2.0 for $C_{1 \mathrm{~b}}, C_{1 \mathrm{p}}$ and $C_{2}$ respectively from [11]. $W_{\min }$ and $W_{\max }$ are taken as 0.1 and 1.0, respectively. The population size of the proposed EPSO has been taken as 10 for case study 1 and 20 for case study 2 . The maximum iterations are set at 500 for all test cases. The proposed algorithm has been developed using MATLAB and simulations have been carried on a personal computer of Intel i5, 3.2 GHz, and $4 \mathrm{~GB}$ RAM and the
Table 1. Optimizing dynamic acceleration coefficient $\mu_{2}$

\begin{tabular}{c|c|c}
\hline$k_{e}$ & $\mu_{2}$ & Average fuel cost (\$) \\
\hline 0.1 & 1.8822 & 166495.262834 \\
\hline 0.2 & 3.9617 & 165982.033125 \\
\hline 0.3 & 5.1781 & 168389.655323 \\
\hline 0.4 & 6.0411 & 168599.160614 \\
\hline 0.5 & 6.7105 & 168689.979717 \\
\hline
\end{tabular}

results obtained after 100 trails are compared with some recent published work.

In the present work, the coefficient of exponent $\mu_{1}$ is assumed to be 5 , as beyond 5 , the term $e^{-\mu}{ }_{1}{ }^{2}$ is not perceptible at the end of search. Further, it has been found through simulations that most appropriate value of $\eta_{t}$ is $2 / 3$. For this value of $\eta_{t}$, the optimized value of $k_{\mathrm{e}}$ is 0.2 and corresponding value of $\mu_{2}$, is 3.9617 as shown in Table 1 . The table presents test results on the basis of average fuel cost obtained after 100 trials of EPSO.

\subsection{Case study 1}

For this case study, a hydrothermal system consisting of a multi-chain cascaded four reservoir hydro plants and a composite thermal plant is considered. The detail data for this system may be referred from [17] and [21]. The solution quality obtained using the proposed EPSO is compared in Table 2 with existing stochastic based techniques published in recent past.

It can be observed from the table that the proposed method yields superior results than the existing methods. Moreover, it provides the best statistical properties such as worst, average and best fuel cost. However, the time of the

Table 2. Comparison results-case study 1

\begin{tabular}{c|c|c|c|c}
\hline Methods & $\begin{array}{c}\text { Best fuel cost } \\
(\$)\end{array}$ & $\begin{array}{c}\text { Average fuel } \\
\text { cost }(\$)\end{array}$ & $\begin{array}{c}\text { Worst fuel } \\
\text { Cost }(\$)\end{array}$ & $\begin{array}{c}\text { CPU } \\
\text { time (s) }\end{array}$ \\
\hline EPSO [22] & 922904.0 & 923527.0 & 924808.0 & - \\
\hline DRQEA [23] & 922526.7 & 923419.4 & 925871.5 & 7.98 \\
\hline CRQEA [17] & 922477.1 & - & - & - \\
\hline SPPSO[20] & 922336.3 & 922668.5 & 923083.5 & 16.3 \\
\hline MAPSO[2] & 922421.0 & 922544.0 & 923508.0 & \\
\hline MHDE [21] & 921893.9 & - & - & - \\
\hline DE [24] & 914343.0 & - & - & - \\
\hline DE-SQP [24] & 912267.9 & - & - & - \\
\hline EPSO & 873164.2 & 880570.0 & 908265.2 & 18.0 \\
\hline
\end{tabular}

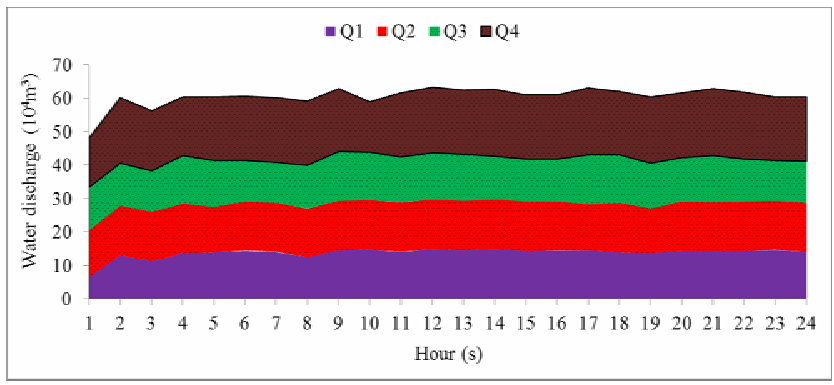

Fig. 2. Optimal value of water discharge for case study 1 
Table 3. Optimal hydrothermal power outputs - case study 1

\begin{tabular}{c|c|c|c|c|c}
\hline \multirow{2}{*}{ Hr. } & \multicolumn{4}{|c|}{$\begin{array}{c}\text { Hydro power generation } \\
(\mathrm{MW})\end{array}$} & $\begin{array}{c}\text { Thermal generation } \\
(\mathrm{MW})\end{array}$ \\
\cline { 2 - 6 } & $P_{\mathrm{h} 1}$ & $P_{\mathrm{h} 2}$ & $P_{\mathrm{h} 3}$ & $P_{\mathrm{h} 4}$ & $P_{\mathrm{s} 1}$ \\
\hline 1 & 65.89 & 86.77 & 58.93 & 227.19 & 931.21 \\
\hline 2 & 96.79 & 72.50 & 57.52 & 301.82 & 861.36 \\
\hline 3 & 81.67 & 75.92 & 58.55 & 263.56 & 880.30 \\
\hline 4 & 110.62 & 72.36 & 36.44 & 279.64 & 790.93 \\
\hline 5 & 110.62 & 90.07 & 64.98 & 299.36 & 724.97 \\
\hline 6 & 111.58 & 111.02 & 60.85 & 301.79 & 824.76 \\
\hline 7 & 110.63 & 110.81 & 52.42 & 303.32 & 1072.83 \\
\hline 8 & 98.83 & 72.13 & 47.54 & 296.71 & 1484.79 \\
\hline 9 & 106.62 & 91.57 & 35.60 & 255.36 & 1750.85 \\
\hline 10 & 86.64 & 111.09 & 36.56 & 176.59 & 1909.13 \\
\hline 11 & 111.37 & 101.80 & 64.73 & 187.29 & 1764.80 \\
\hline 12 & 113.50 & 100.14 & 64.75 & 304.03 & 1727.58 \\
\hline 13 & 103.99 & 94.86 & 62.53 & 269.34 & 1699.28 \\
\hline 14 & 113.50 & 72.44 & 38.17 & 306.00 & 1669.89 \\
\hline 15 & 107.01 & 72.50 & 47.33 & 186.91 & 1716.25 \\
\hline 16 & 86.61 & 95.13 & 38.43 & 282.20 & 1567.63 \\
\hline 17 & 109.69 & 97.04 & 35.88 & 256.42 & 1630.98 \\
\hline 18 & 93.23 & 104.05 & 54.82 & 185.36 & 1702.54 \\
\hline 19 & 110.57 & 107.26 & 37.68 & 305.08 & 1679.41 \\
\hline 20 & 112.06 & 111.11 & 37.91 & 300.04 & 1718.88 \\
\hline 21 & 86.56 & 110.93 & 65.01 & 305.78 & 1671.71 \\
\hline 22 & 104.75 & 92.79 & 56.75 & 204.13 & 1661.57 \\
\hline 23 & 110.59 & 111.20 & 62.02 & 185.84 & 1380.35 \\
\hline 24 & 111.75 & 110.77 & 38.52 & 274.20 & 1054.76 \\
\hline
\end{tabular}

EPSO is slightly higher than other methods due to obvious reasons. The optimal value of hydro discharges $\left(10^{4} \mathrm{~m}^{3}\right)$ is given in Fig. 2. The obtained optimal values of hydro and thermal generations are shown in Table 3. It can be verified from the table that the proposed algorithm accurately handles all hydrothermal constraints.

\subsection{Case study 2}

In order to validate the performance of the proposed EPSO on large dimensional system, it has been applied to a power system consisting of four hydro and ten thermal units. The data for this system may be referred [20, 25]. The results obtained using the proposed EPSO is compared with other existing stochastic based techniques in Table 4. The table shows that the proposed method gives substantially better results than existing methods. Moreover, it may also be seen from the table that the proposed EPSO produces statistically better quality solution in terms of best, average and worst fuel cost. This shows that the proposed method

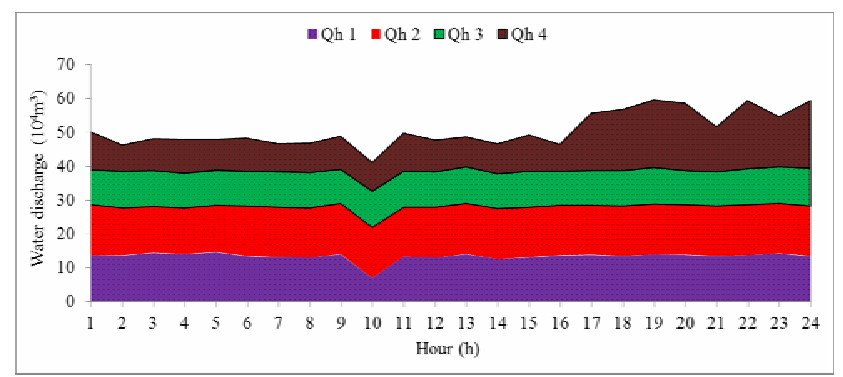

Fig. 3. Optimal value of water discharge for case study 2 is consistent and robust. The CPU time is also much less than other existing stochastic methods except SPPSO [20]. The optimal values of water discharges and hydrothermal scheduling are plotted in Fig. 3 and 4. The detailed optimal generating schedules of thermal plants for this system are given in Table 5 and 6.

Any stochastic based search technique must be designed to accomplish global exploration (searching new areas) and must tend to facilitate local exploitation (fine tuning of the current solution). In order to highlight this feature of the proposed method a comparison of the convergence

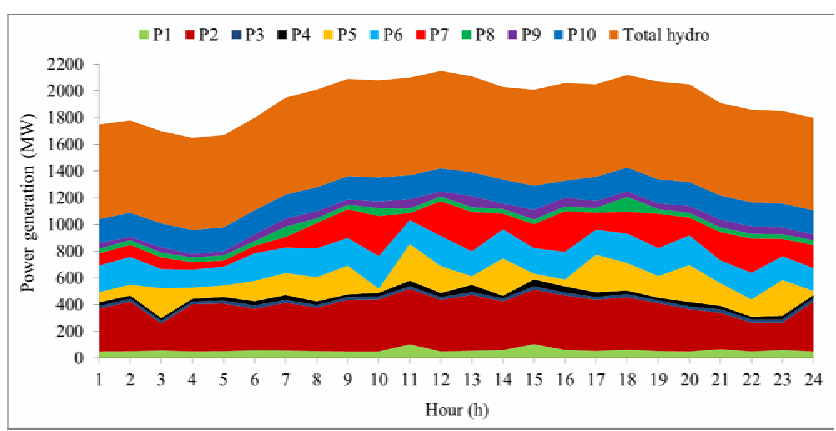

Fig. 4. Optimal value of power generation for case study 2

Table 4. Comparison results - case study 2

\begin{tabular}{c|c|c|c|c}
\hline Methods & $\begin{array}{c}\text { Best cost } \\
(\$)\end{array}$ & $\begin{array}{c}\text { Average cost } \\
(\$)\end{array}$ & $\begin{array}{c}\text { Worst cost } \\
(\$)\end{array}$ & $\begin{array}{c}\text { CPU time } \\
(\mathrm{s})\end{array}$ \\
\hline DE [25] & 170964.2 & - & - & 96.4 \\
\hline MDE [20] & 177338.6 & 179676.4 & 182172.0 & 86.5 \\
\hline SPSO [20] & 189350.6 & 190560.3 & 191844.3 & 108 \\
\hline SPPSO [20] & 167710.6 & 168688.9 & 170879.3 & 24.8 \\
\hline EPSO & 161513.7 & 165982.0 & 169885.3 & 33.7 \\
\hline
\end{tabular}

Table 5. Optimal thermal power outputs - case study 2

\begin{tabular}{c|c|c|c|c|c}
\hline \multirow{2}{*}{ Hr. } & \multicolumn{5}{|c}{ Thermal generation $(\mathrm{MW})$} \\
\cline { 2 - 6 } & $P_{\mathrm{s} 1}$ & $P_{\mathrm{s} 2}$ & $P_{\mathrm{s} 3}$ & $P_{\mathrm{s} 4}$ & $P_{\mathrm{s} 5}$ \\
\hline 1 & 50.0000 & 319.9945 & 20.6322 & 27.4205 & 76.0680 \\
\hline 2 & 51.6748 & 371.8779 & 20.8890 & 24.0137 & 81.4950 \\
\hline 3 & 56.7854 & 202.6099 & 20.0000 & 20.4599 & 223.1605 \\
\hline 4 & 50.2463 & 353.6806 & 20.2463 & 20.2463 & 83.2259 \\
\hline 5 & 52.1957 & 356.4214 & 22.0409 & 27.2309 & 86.1987 \\
\hline 6 & 57.9212 & 311.2400 & 26.0838 & 31.6403 & 151.2828 \\
\hline 7 & 56.3045 & 358.4581 & 20.9470 & 35.8899 & 165.8324 \\
\hline 8 & 52.3533 & 325.4259 & 20.0677 & 27.4512 & 180.7397 \\
\hline 9 & 50.0000 & 387.1642 & 20.0000 & 20.0000 & 214.9171 \\
\hline 10 & 50.2272 & 389.7312 & 21.3417 & 27.5668 & 30.6048 \\
\hline 11 & 102.4214 & 415.9246 & 20.0000 & 41.0229 & 274.8453 \\
\hline 12 & 50.0000 & 386.9061 & 20.0000 & 30.5679 & 201.6383 \\
\hline 13 & 55.3415 & 417.4069 & 22.4414 & 53.5951 & 64.0058 \\
\hline 14 & 59.8128 & 365.4930 & 21.4507 & 20.0000 & 279.7595 \\
\hline 15 & 104.1482 & 408.1953 & 25.5453 & 51.4825 & 43.5571 \\
\hline 16 & 61.2054 & 407.5337 & 21.7766 & 46.2230 & 52.0867 \\
\hline 17 & 53.7361 & 383.8773 & 20.2449 & 34.9254 & 282.2489 \\
\hline 18 & 62.7855 & 391.8634 & 24.7498 & 26.1295 & 206.6527 \\
\hline 19 & 52.8118 & 359.0870 & 20.0973 & 20.0000 & 162.0745 \\
\hline 20 & 50.0000 & 315.5971 & 21.4181 & 33.6181 & 276.6590 \\
\hline 21 & 66.7122 & 271.9004 & 26.4579 & 26.0372 & 169.4507 \\
\hline 22 & 50.2739 & 213.9358 & 24.9465 & 20.2739 & 129.9473 \\
\hline 23 & 60.0837 & 204.7673 & 25.3240 & 26.8018 & 267.5672 \\
\hline 24 & 50.0727 & 374.7331 & 23.3773 & 24.0544 & 29.8815 \\
\hline & & & & &
\end{tabular}


Table 6. Optimal thermal power outputs - case study 2

\begin{tabular}{c|c|c|c|c|c}
\hline \multirow{2}{*}{ Hr. } & \multicolumn{5}{|c}{ Thermal generation $(\mathrm{MW})$} \\
\cline { 2 - 6 } & $P_{\mathrm{s} 6}$ & $P_{\mathrm{s} 7}$ & $P_{\mathrm{s} 8}$ & $P_{\mathrm{s} 9}$ & $P_{\mathrm{s} 10}$ \\
\hline 1 & 200.1175 & 89.6157 & 35.0000 & 42.7840 & 179.768 \\
\hline 2 & 208.0039 & 90.3282 & 35.3181 & 25.3181 & 180.000 \\
\hline 3 & 143.9131 & 87.1887 & 35.0000 & 41.4158 & 178.847 \\
\hline 4 & 135.0155 & 55.0076 & 36.4701 & 25.2463 & 180.000 \\
\hline 5 & 140.8319 & 45.7434 & 38.5172 & 29.8460 & 180.000 \\
\hline 6 & 207.2802 & 55.5748 & 35.5437 & 51.0575 & 180.000 \\
\hline 7 & 190.5979 & 79.3704 & 77.1129 & 60.7295 & 180.000 \\
\hline 8 & 216.3585 & 189.4526 & 35.0000 & 53.2236 & 178.900 \\
\hline 9 & 207.5738 & 214.7833 & 35.0000 & 38.5354 & 174.908 \\
\hline 10 & 244.4784 & 300.0667 & 59.1068 & 49.9829 & 180.000 \\
\hline 11 & 178.9240 & 53.0306 & 35.0000 & 69.2647 & 178.916 \\
\hline 12 & 225.4786 & 261.5538 & 35.0000 & 37.5061 & 172.757 \\
\hline 13 & 186.7890 & 294.9863 & 35.7279 & 82.7075 & 180.000 \\
\hline 14 & 217.8534 & 117.9779 & 35.0000 & 40.2093 & 179.962 \\
\hline 15 & 191.1471 & 179.0280 & 35.2079 & 73.2517 & 180.000 \\
\hline 16 & 207.3996 & 304.3836 & 35.0000 & 66.3182 & 126.874 \\
\hline 17 & 186.5666 & 127.4671 & 35.2449 & 53.3937 & 180.000 \\
\hline 18 & 220.9733 & 163.2244 & 111.5732 & 39.5480 & 180.000 \\
\hline 19 & 209.0477 & 257.8150 & 35.0000 & 43.5913 & 179.276 \\
\hline 20 & 221.9356 & 133.4587 & 35.0000 & 51.7964 & 179.317 \\
\hline 21 & 170.7584 & 212.2499 & 35.1598 & 58.7733 & 180.000 \\
\hline 22 & 199.0818 & 257.0421 & 35.2739 & 56.7248 & 180.000 \\
\hline 23 & 178.3044 & 129.7854 & 35.4487 & 49.5290 & 180.000 \\
\hline 24 & 171.9208 & 173.2590 & 35.0727 & 45.1577 & 180.000 \\
\hline & & & & &
\end{tabular}

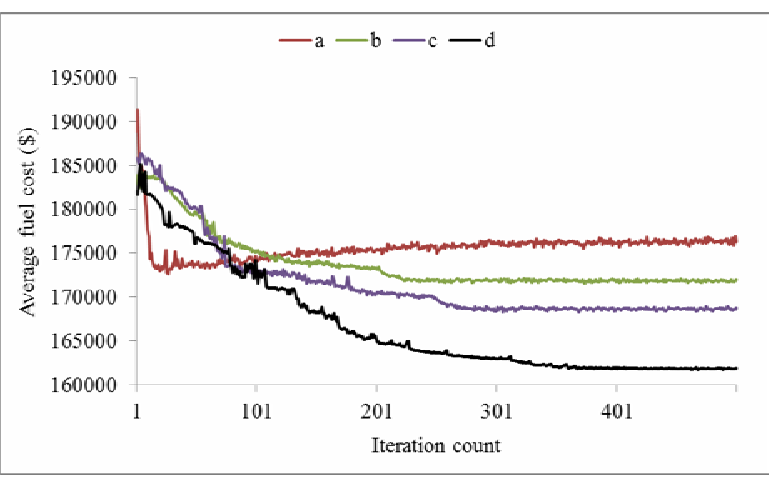

Fig. 5. Convergence characteristic for PSO and EPSO

characteristics for average and best fuel cost for the Case study 2 are shown in Fig. 5 and Fig. 6.

In these figures, the characteristic ' $a$ ' stands for the conventional PSO, ' $b$ ' refers to ' $a$ ' with exponential modulation in inertia weight, 'c' refers to ' $b$ ' with improved cognitive behavior due to preceding experience and ' $d$ ' refers to ' $c$ ' with constriction functions i.e. proposed EPSO. It is evident from the Fig. 5 that the conventional PSO is very quickly trapped in local optima. It can also be observed from the figure that the performance of PSO is marginally improved when inertia weight is exponentially modulated and is further improved when preceding experience of particles is also considered. However, these two modifications are not sufficient to exploit the promising region effectively and efficiently. This leads to premature convergence due to local trappings. It happens because in all above mentioned versions of PSO, particles flies to the promising region very early and thus

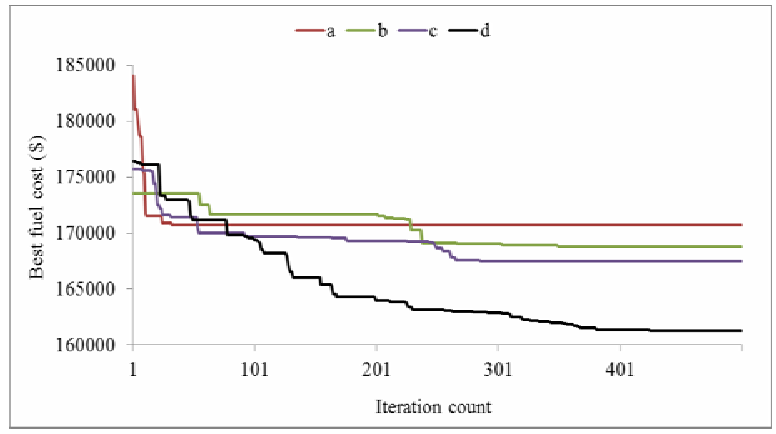

Fig. 6. Convergence characteristic for PSO and EPSO

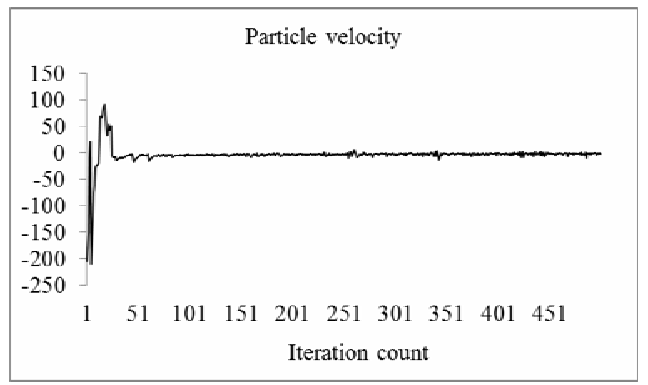

Fig. 7. Particle's velocity in PSO

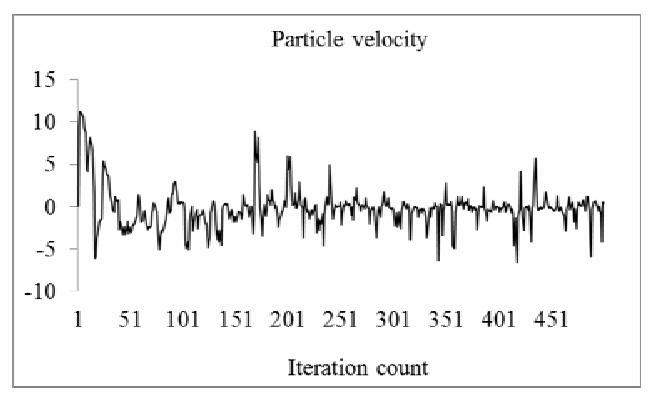

Fig. 8. Particle's velocity in EPSO

probably missing the global or near global optima owing to higher assigned velocities.

In EPSO, the proposed constriction functions regulate particles' velocities so that they fly more comprehensively in the search space during their whole journey. Thus it avoids local optima several times before approaching in the promising region. In fact, during the initial phase of EPSO, particles enable to explore wider search space owing to higher initial cognitive component than the social component. This helps in identifying the region of global optima accurately. However, towards the latter half of the search, all particles move with strong communication and intensively exploit the region near the global optima owing to relatively strong social component. As a result, all particles are diverted towards the global optima near the end of the search. Similar conclusions can be drawn from Fig. 6 showing convergence characteristic for the best fuel cost of a sample trial.

In order to appreciate and understand the performance of 


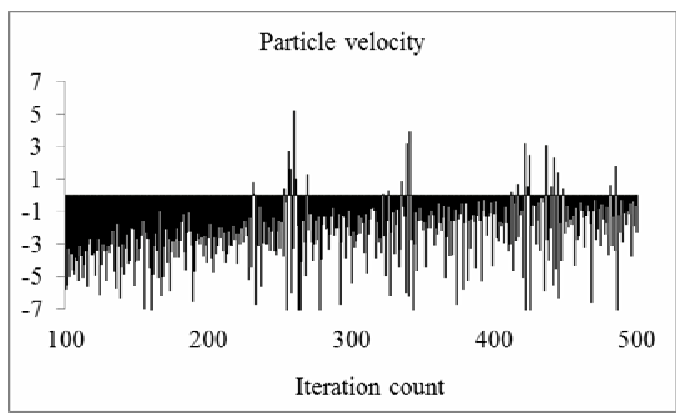

Fig. 9. Enlarged view of particle's velocity in PSO

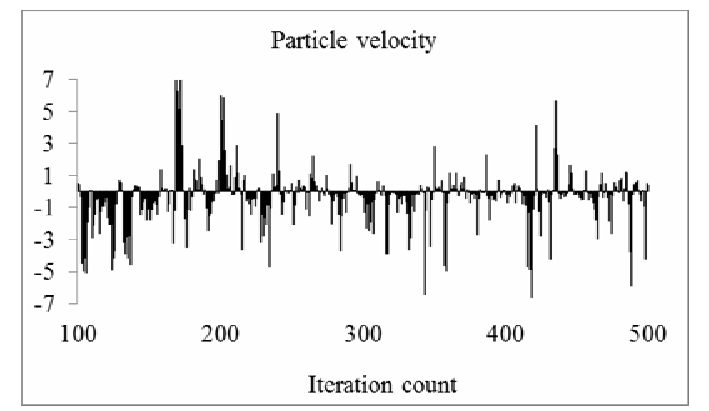

Fig. 10. Enlarged view of particle's velocity in EPSO

the proposed method, a comparison of the movement of a sample particle in the conventional and proposed PSO is illustrated in Fig. 7 and 8, repectively. The comparison of Fig. 7 with Fig. 8 reveals that particle moves with very high velocity in PSO during initial iterations, whereas it moves with moderate velocity in EPSO. Moreover,from the Fig. 9 and 10 it may be observed that in EPSO particles have both negative and positive excursions of velocity, which is almost unidirectional in case of conventional PSO. Therefore, better exploration and exploitation of search space is achieved in EPSO.

\section{Conclusion}

The STHS problem is a highly complex, nonlinear, nonconvex, hard combinatorial optimization problem satisfying several equality and inequality constraints. This paper presents an Enhanced PSO to solve STHS problem and attempts to overcome some drawbacks of existing PSO methods. The proposed method has been tested to solve STHS problems of two different test systems of different dimensions with a variety of operational and network constraints. The application results are also compared with existing PSO and other swarm intelligence based methods. The application results show that the proposed method is efficient and is usually not trapped in local minima. The comparison shows that the proposed EPSO method is capable of giving better results than the existing PSO and other stochastic based methods. This may be due to the fact that EPSO essentially aims to regulate particle velocity during its whole course of flight in such a fashion so as to enhance exploration and exploitation capabilities of the PSO. It is noteworthy that the proposed EPSO is free from any additional mechanism to avoid local trapping, empirical formulae to bound particle's velocity or squeezing search space. Moreover, the proposed algorithm is robust as it generates better quality solutions irrespective of the initial position of the particles. The proposed EPSO can be extended to solve STHS problems with the inclusion of more objectives and constraints like environmental issues, reserve capacity, network security, network congestion management, etc.

\section{Acknowledgements}

The authors would like to thank the editor and reviewers for their valuable comments and recommendations.

\section{References}

[1] V. Senthil Kumar and M. R. Mohan, "A genetic algorithm solution to the optimal short-term hydrothermal scheduling," Electrical Power and Energy Systems, vol. 33, pp. 827-835, 2011.

[2] N. Amjady and H. R. Soleymanpour, "Daily hydrothermal generation scheduling by a new modified adaptive particle swarm optimization technique," Electric Power Systems Research, vol. 80, pp.723-732, 2010.

[3] J. Kennedy and R. Eberhart, Swarm intelligence, Morgan Kaufmann Publishers, 2001.

[4] D. N. Jeyakumar, T. Jayabarathi, and T. Raghunathan, "Particle swarm optimization for various types of economic dispatch problems," Electrical Power and Energy Systems, vol. 28, pp. 36-42, 2006.

[5] A. Safari and H. Shayeghi, "Iteration particle swarm optimization procedure for economic load dispatch with generator constraints," Expert Systems with Applications, vol. 38, pp. 6043-6048, 2011.

[6] J. B. Park, Y.-W. Jeong, J.R. Shin and K. Y. Lee, “An improved particle swarm optimization for non-convex economic dispatch problems," IEEE Trans. Power Syst., vol. 25, no. 1, pp. 156-166, Feb. 2010.

[7] T. Niknam, H. D. Mojarrad, and H. Z. Meymand, "Non-smooth economic dispatch computation by fuzzy and self adaptive particle swarm optimization," Applied Soft Computing, vol. 11, pp. 2805-2817, 2011.

[8] B. Yu, X. Yuan, and J. Wang, "Short-term hydrothermal scheduling using particle swarm optimization method," Energy Convers and Manage, vol. 48, no. 7, pp. 1902-8, 2007.

[9] G. Baskar and M. R. Mohan, "Security constrained economic load dispatch using improved particle swarm optimization suitable for utility system," 
Electrical Power and Energy Systems, vol. 30, pp. 609-613, 2008.

[10] L. Wang, and C. Singh, "Stochastic economic emission load dispatch through a modified particle swarm optimization algorithm," Electric Power Systems Research, vol. 78, pp. 1466-1476, 2008.

[11] A. I. Selvakumar and K. Thanushkodi, "A new particle swarm optimization solution to non-convex economic dispatch problems," IEEE Trans. Power Syst., vol. 22, no. 1, pp. 42-51, Feb. 2007.

[12] R. Roy and S.P. Ghoshal, "A novel crazy swarm optimized economic load dispatch for various types of cost functions," Electrical Power and Energy Systems, vol. 30, pp. 242-253, 2008.

[13] A. Ratnaweera, S. K. Halgamuge, and H. C. Watson, "Self-Organizing Hierarchical Particle Swarm Optimizer with Time-Varying Acceleration Coefficients," IEEE Transactions on Evolutionary Computation, vol. 8, no. 3, pp. 240- 255, June 2004.

[14] K. T. Chaturvedi, M. Pandit and L.Srivastava, "Particle swarm optimization with time varying acceleration coefficients for non-convex economic power dispatch," Electrical Power and Energy Systems, vol. 31, pp. 249-257, 2009.

[15] K. K. Mandal and N. Chakraborty, "Daily combined economic emission scheduling of hydrothermal systems with cascaded reservoirs using self-organizing hierarchical particle swarm optimization technique," Expert Systems with Applications, vol. 39, pp. 34383445, 2012.

[16] B. M. Ivatloo, "Combined heat and power economic dispatch problem solution using particle swarm optimization with time varying acceleration coefficients," Electric Power Systems Research, vol. 95, pp. 9- 18, 2013.

[17] Y. Wang, J. Zhou, L. Mo, S. Ouyang, and Y Zhang “A clonal real-coded quantum-inspired evolutionary algorithm with Cauchy mutation for short-term hydrothermal generation scheduling," Electrical Power and Energy Systems, vol. 43, pp.1228-1240, 2012.

[18] Y. Shi and R. C. Eberhart, "Empirical study of particle swarm optimization," in Proc. 1999 IEEE Congress on Evolutionary Computation, Piscataway, NJ, USA, IEEE Press, pp. 1945-1950.

[19] L. D. S. Coelho and C. S. Lee, "Solving economic load dispatch problems in power systems using chaotic and gaussian particle swarm optimization approaches," Electrical Power and Energy Systems, vol. 30, pp. 297-307, 2008.

[20] J. Zhang, J. Wang, and C. Yue, "Small PopulationBased Particle Swarm Optimization for Short-Term Hydrothermal Scheduling," IEEE Transactions on Power Systems, vol. 27, no. 1, pp. 142-152, Feb. 2012.

[21] L. Lakshminarasimman, and S. Subramanian, "A modified hybrid differential evolution for short-term scheduling of hydrothermal power systems with cascaded reservoirs," Energy Conversion and Management, vol. 49, pp. 2513-2521, 2008.

[22] X. Yuan, L. Wang, and Yanbin Yuan, “Application of enhanced PSO approach to optimal scheduling of hydro system," Energy Conversion and Management, vol. 49, pp. 2966-2972, 2008.

[23] Y. Wang, J. Zhou, L. Mo, R. Zhang, and Y. Zhang, "Short-term hydrothermal generation scheduling using differential real-coded quantum-inspired evolutionary algorithm," Energy, vol. 44, pp. 657-671, 2012.

[24] S. Sivasubramani, and K. Shanti Swarup, "Hybrid DE-SQP algorithm for non-convex short term hydrothermal scheduling problem," Energy Conversion and Management, vol. 52, pp. 757-761, 2011.

[25] K. K. Mandal, and N. Chakraborty, "Differential evolution technique-based short-term economic generation scheduling of hydrothermal systems," Electric Power Systems Research, vol. 78, pp.19721979, 2008.

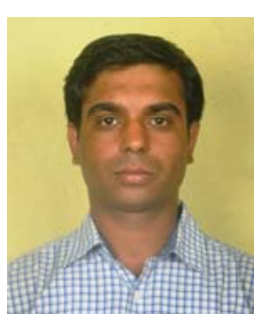

Vinay Kumar Jadoun has obtained his B. E. (Electrical Engineering) in 2007 from Samrat Ashok Technological Institute (SATI), Vidisha, M. P., India and M. Tech. (Power System) in 2010 from Electrical Engineering Department of Malaviya National Institute of Technology, Jaipur, India.

$\mathrm{He}$ is currently a $\mathrm{Ph}$. D. research scholar in the Electrical Engineering Department of Malaviya National Institute of Technology, Jaipur, India. His areas of interest are economic dispatch, hydrothermal scheduling, multi-area economic dispatch, and soft computing techniques.

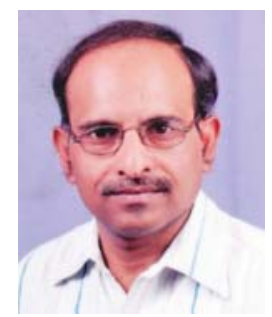

Nikhil Gupta received M. Tech. and Ph.D., both in Electrical Engineering from Malaviya National Institute of Technology, Jaipur, India in 2006 and 2012, respectively. $\mathrm{He}$ is presently working as Assistant Professor in the same institute. His present research interests are the operation and control of power systems and computational intelligence.

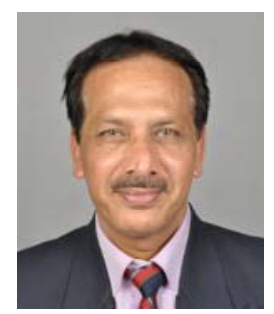

Khaleequr Rehman Niazi has over 25 years of teaching and research experience. Currently he is a Professor in the Department of Electrical Engineering, Malaviya National Institute of Technology Jaipur, India. $\mathrm{He}$ has published over 100 papers in journals and conferences. He has supervised 
several Ph.D. and master's students. He has diversified research interests in the areas of conventional power and renewable energy systems, including power system stability, distribution network reconfiguration, FACTS, and application of artificial intelligence (AI) and artificial neural network (ANN) techniques to power systems.

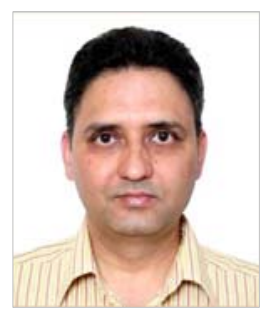

Anil Swarnkar received M. Tech. and Ph.D., both in Electrical Engineering from Malaviya National Institute of Technology, Jaipur, India in 2005 and 2012, respectively. $\mathrm{He}$ is presently working as Assistant Professor in the same institute. His present research interests are the operation and control of power systems and AI techniques. 